\title{
Assessing microscopic and rapid diagnostic tests for malaria among pregnant women with fever in Ondo State, Southwest Nigeria
}

\begin{abstract}
Background: Malaria as a health burden in sub-Saharan Africa is well-known. Information about access to programs by pregnant women during early antenatal care (ANC) visits and the impact on the burden of malaria is limited.

Objective: To evaluate the utilization of malaria control services among pregnant women with fever between 2014 and 2018 in Ondo State, Southwest Nigeria.

Materials and methods: Between 2014 and 2018, malaria-related indicators including the proportion of pregnant women with fever who were investigated with microscopy and Rapid Diagnostic Test (RDT) were tracked in Ondo State, Nigeria. Data were captured using the District Health Information Management version 2.0 (DHISv2.0) and analyzed using Stata 13 statistical software.

Results: Throughout the study period, the mean $( \pm \mathrm{sd})$ percentage of pregnant women with fever tested for malaria parasites with microscopy in all the LGAs was 2.71 (4.87) with the lowest and highest means ( \pm sd) occurring in 2016 as $1.20(1.71)$ and in 2018 as 5.47 (7.93) respectively with no significant difference $(\mathrm{F}$-statistics $=2.25 ; \mathrm{P}$-value $=0.07) . \mathrm{A}$ statistically significant variation $(\mathrm{F}$-statistics $=34.90$; $\mathrm{P}$-value $=0.0000001)$ was observed in the overall mean $( \pm \mathrm{sd})$ proportion of pregnant women with fever who were tested for malaria with RDT over the study period with the lowest as 57.0 (15.2) in 2014 and the highest as 96.2 (4.1) in 2017.This level of significance was reflected mostly in the coastal (F-statistics $=15.05 ; \mathrm{P}$-value $=0.0000001)$ and in the rainforest $(\mathrm{F}$-statistics $=12.86$; $\mathrm{P}$-value $=0.0000001)$ ecological zones of the state but much less in the Savannah ecological zone (F-statistics=3.23; P-value=0.04). There was no significant correlation (Pearson's $\mathrm{r}=0.160$, P-value $=0.141$ ) between fever cases that tested positive using microscopy and fever cases that tested positive with RDT. A noteworthy variation (F-statistics $=3.96$, $\mathrm{P}$-value $=0.005$ ) was observed in the proportion of fever cases that tested positive with RDT but not with microscopy. Overall mean $( \pm$ sd) proportion of pregnant women with confirmed fever cases was 76.66 (15.06), mostly in the Savannah ecological system $(81.84 \pm 21.18)$ throughout the study period.

Conclusion: Data from this study suggests that RDT gradually replaced microscopy in testing for malaria among pregnant women in Ondo State of Nigeria. It also suggests that some ecological zones may be more underserved than others in assessing malaria in pregnancy. More technical and financial assistance are needed for the State Malaria Elimination Program to improve ANC service utilization.
\end{abstract}

Keywords: malaria in pregnancy, malaria microscopy, RDT, ecological zones of Ondo State
Volume II Issue 3 - 2020

\author{
Waheed Folayan,' Titilola MAfolabi, ${ }^{2}$ Oladipo \\ B Akinmoladun,' Feyijimi Egunjobi,' Lynda \\ Ozor, ${ }^{3}$ Tolulope Fagbemi, ${ }^{4}$ Bamgboye $M$ \\ Afolabi $^{5}$ \\ 'Ondo State Ministry of Health, State Malaria Elimination \\ Program, Akure, Ondo State, Nigeria \\ ${ }^{2}$ Midwestern University, Glendale, Arizona, USA \\ ${ }^{3}$ World Health Organization, Malaria Unit,Abuja Office, Nigeria \\ ${ }^{4}$ National Malaria Elimination Program, Abuja Nigeria \\ ${ }^{5} \mathrm{Health}$, Environment and Development Foundation, Lagos, \\ Nigeria
}

Correspondence: Bamgboye M Afolabi, Health, Environment and Development Foundation, 18 Ogunfunmi Street, Lagos, Nigeria, Email bmafolabi@gmail.com

Received: April 24, 2020 | Published: May I I, 2020
Abbreviations: ANC, antenatal care; RDT, rapid diagnostic test; MIP, malaria in pregnancy; WHO, World Health Organization; IPTp, intermittent presumptive treatment in pregnancy; SP, sulphadoxine-pyrimethamine; LGAs, local Government areas; DHIS, District health information system; HPF, high power field; PfHRP-2, plasmodium falciparum histidine rich protein-2; ANOVA, analysis of variance; NHMIS, National health management information system; QA, quality assurance; SOP, standard operating procedure

\section{Introduction}

Malaria in pregnancy (MIP) is a global phenomenon, prevalent in malaria-endemic parts of the world. ${ }^{1}$ In sub-Saharan Africa, malaria exerts the heaviest burden in pregnancy as a leading and direct or indirect cause of maternal and perinatal morbidity and mortality. ${ }^{2-4}$ Studies have indicated that malaria causes fetal problems such as miscarriages, stillbirths, preterm deliveries, ${ }^{5,6}$ neonatal problems such as low birth weight ${ }^{7}$ and maternal dilemma such anemia. ${ }^{8}$ The first pregnancy, especially, is documented as one of the focal risk factors for malaria and anaemia. ${ }^{9-11}$ Majority of indigenous Black African women in sub-Saharan Africa are semi-immune to falciparum malaria indicating that repeated infection with asymptomatic falciparum malaria seldom results in disease severity. ${ }^{12}$ Studies have suggested that the lethal effect of MIP is linked to placental sequstration of plasmodium falciparum malaria, especially in women who were semi- 
immune prior to pregnancy. ${ }^{13}$ In a cohort of women, a large quantity of falciparum malaria parasites are occassionally found sequestrated in the placenta when, simultaneously, none can be found in peripheral blood circulation. ${ }^{14}$ This affinity of falciparum malaria parasites for the human placenta has been suggested to be the link between MIP and subsequent low birthweight. ${ }^{9,15}$ World Health Organization (WHO) recommends prompt parasite-based diagnosis by microscopy or malaria rapid diagnostic test (RDT) in all patients suspected of malaria before antimalarial treatment is administered. ${ }^{16}$ The WHO also recommends giving intermittent presumptive treatment in pregnancy (IPTp) in the form of Sulphadoxine-pyrimethamine (SP) to every pregnant woman irrespective of whether they are found to have malaria parasites in their blood or not. ${ }^{17}$ Given twice or thrice to all pregnant women in highly endemic areas, SP leads to a reduction in maternal anaemia and an increase in birthweight. ${ }^{18-22}$ As in our previous study, ${ }^{23}$ dataset from Ondo State Malaria Elimination Program Demographic and Health Information System 2014 to 2018 was analyzed with the objective of identifying possible disparities in the utilization of antenatal care (ANC) services, especially testing for malaria parasitaemia using microscopy and RDT among pregnant women in the Local Government Areas (LGAs) and in various ecological zones within the state.

\section{Materials and methods}

Malaria microscopy is the gold standard for detecting not only presence of parasites but also for calculating parasite density and describing the species and stage of development of the parasites. Rapid Diagnostic Tests (RDTs) are antigen-based method for detecting recent malaria infection as described earlier. ${ }^{24}$
Study design: The study design was a comparative, retrospective cohort review of data extracted from District Health Information System 2.0 (DHIS) between 2014 and 2018, comprising of information of pregnant women's visits to ANC facilities throughout the state. The data considered in this paper are the proportion of pregnant women who

a. Presented with fever of $\geq 37.5^{\circ} \mathrm{C}$, suspected to be malaria and were (a) tested with microscopy and RDT

b. Positive with microscopy or with RDT

c. Confirmed uncomplicated malaria cases. Microscopy results were compared with RDT data from pregnant women in the state.

Study area: The study area, study population and infrastructure have already been reported in a previous publication. ${ }^{23}$ Briefly, Ondo State is located in the Southwest geo-political zone of Nigeria (Figure 1), bordered by Ekiti and Kogi States in the north, Edo State to its east, Ogun and Osun States on the southwest and the Atlantic Ocean to the south. From the southern part of the state to the north, there are three distinctly demarcated ecological zones - Atlantic Ocean Coastline, Rainforest and Savannah - each with specific number of local government areas (LGAs) as earlier reported. Briefly, these are

i. Atlantic Ocean Coastline-Ilaje, Ese-Odo, Okiti-pupa and Irele

ii. Rainforest - Odigbo, Ondo West, Ondo East, Idanre, Ose, Owo, Akure North, Akure South, Ifedore and Ile-Oluji/Okeigbo and

iii. Savannah - Akoko Southwest, Akoko Southeast, Akoko Northwest and Akoko Northeast.

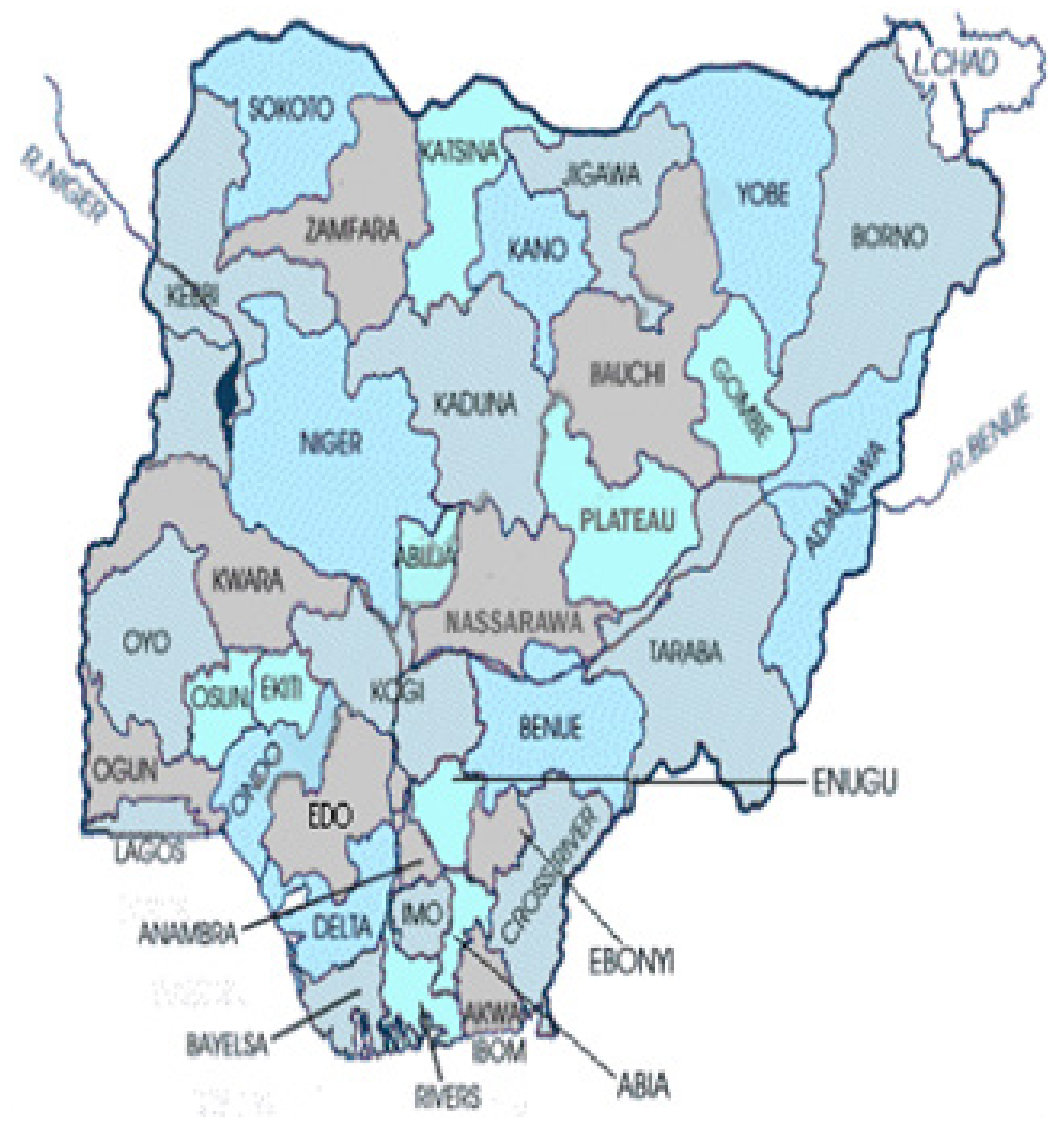

Citation: Folayan W,Afolabi TM,Akinmoladun OB, et al.Assessing microscopic and rapid diagnostic tests for malaria among pregnant women with fever in Ondo State, Southwest Nigeria. Obstet Gynecol Int J. 2020; I I (3): I 43-I53. DOI: I0.15406/ogij.2020.I I.0050 I 


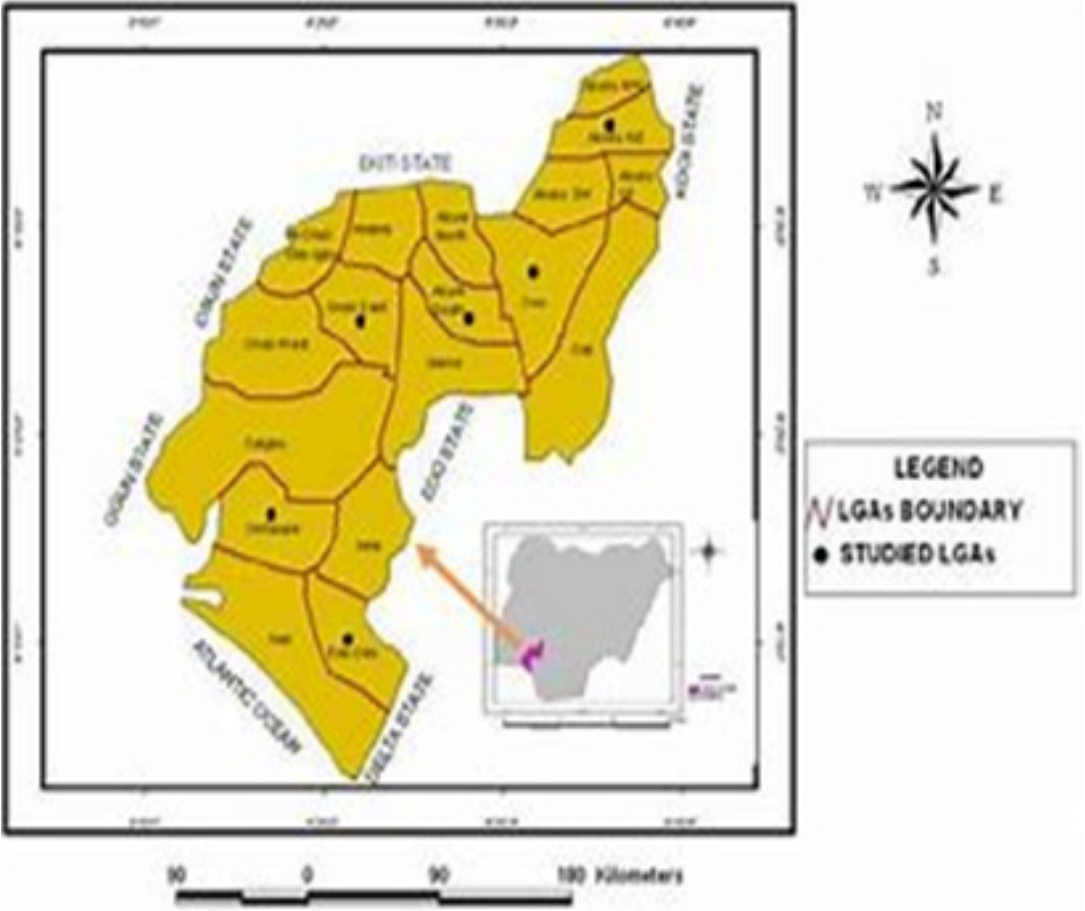

Figure I Map of Nigeria (https://www.ngex.com/nigeria/places/default.htm) and composite map of Ondo State (https://www.researchgate.net/).

The data flow from community to LGAs and to DHIS, sampling, inclusion and exclusion criteria and data management have already been described. ${ }^{23}$ In brief, data for all ANC visits in the state from 2014 to 2018 was retrieved. All public health facilities in the state were included in the study. Data from private and from Faith-based health facilities were excluded from the study. The data included multiple visits by pregnant women, especially when these pregnant women complained of fever.

Microscopy: The state used WHO protocol ${ }^{25}$ in preparing thick and thin blood films. Briefly, after the blood films were prepared, the thin smears were fixed with methanol and the slides were allowed to air dry until the following day when they were stained for about 30 minutes with $3 \%$ Giemsa and examined under 100X (high power field $[\mathrm{HPF}]$ ) oil immersion lenses. Under the assumption that $5-8 \mu \mathrm{L}$ of blood was used in preparing a thick blood film and that $0.002 \mu \mathrm{L}$ of blood was in an $\mathrm{HPF}^{26}$ parasite density ( $/ \mu \mathrm{L}$ of blood) was calculated by multiplying the number of parasites per HPF by 500 . At least 200 HPFs were read to declare a slide negative.

Rapid Diagnostic Test (RDT): This tests for the Plasmodium falciparum histidine rich protein-2 (PfHRP-2), a water-soluble parasite-specific protein released from parasite-infected erythrocytes.

Outcomes measured: Outcomes were based on the proportion of pregnant women with self-reported fever who were tested for malaria using microscopy or RDT, in whom uncomplicated malaria was confirmed. To evaluate, the proportions of pregnant women tested for malaria parasites using microscopy or RDT and the proportion with uncomplicated malaria were disaggregated according to local government areas and ecological zones before analysis.

Definitions: Fever was defined as body temperature of $\geq 37.5^{\circ} \mathrm{C}$.
Management of confirmed malaria in pregnancy: All pregnant women who presented with fever and were malaria positive were treated with government-approved protocol for managing malaria in pregnancy.

Statistical analysis: Data were first entered into Excel sheet where it was cleaned and coded before analysis using Stata-13 statistical software. The mean $( \pm \mathrm{sd})$ and various rates were determined for continuous variables. Students t-test and chi square analysis were used to compare differences of two variables and Analysis of Variance (ANOVA) for more than two variables. Differences of means were considered significant at a $95 \%$ confidence limit when P-values were below 0.05 . Association was determined by computing logistic regression analysis of LGAs with other exposure variables at $95 \%$ confidence interval. Data were presented as Tables and graphs.

\section{Results}

Data reported in this study were generated between 2014 and 2018 as part of National Health Management Information System (NHMIS) of Nigeria with emphasis on Ondo State, Southwest of the country.

\section{Mean proportion of pregnant women with fever and uncomplicated malaria cases tested with microscopy or RDT in all LGAs and in ecological zones}

Analysis of variance for the mean proportion of pregnant women with fever tested by microscopy and RDT, confirmed for malaria parasitaemia by microscopy or RDT in all the LGAs or ecological zones of the state throughout the study period are reported in Table 1. Only fever cases tested with RDT (F-statistics $=34.9, \mathrm{P}$-value $=0.0000001$ ), fever cases that tested positive for malaria with RDT (F-statistics $=3.96$, $\mathrm{P}$-value $=0.005)$ and confirmed uncomplicated malaria cases (F-statistics $=7.80$, P-value $=0.0000001)$ had statistically significant 
values. Additionally, the mean number of pregnant women with fever cases tested with microscopy, was significantly different only in the rain forest ecological zone (F-statistics $=2.59$, P-value $=0.049$ ). Apart from fever cases tested with RDT, there were no significant variations in all other variables of interest in the Coastal and in the Savannah

Table I Analysis of variance for mean proportion of pregnant women with fever and other variables tested with microscopy or in all LGAs and in ecological zones of Ondo State 2014-2018

\begin{tabular}{|c|c|c|c|c|c|c|c|c|c|c|}
\hline \multirow{3}{*}{ Variable } & \multirow{2}{*}{\multicolumn{2}{|c|}{$\begin{array}{l}\text { LGAs } \\
\text { All }\end{array}$}} & \multicolumn{8}{|c|}{ Ecological zone } \\
\hline & & & \multicolumn{2}{|l|}{ All } & \multicolumn{2}{|l|}{ Coastal } & \multicolumn{2}{|l|}{ Rain forest } & \multicolumn{2}{|l|}{ Savannah } \\
\hline & F-statistic & P-value & F-statistic & P-value & F-statistic & P-value & F-statistic & P-value & F-statistic & P-value \\
\hline $\begin{array}{l}\text { Fever cases } \\
\text { tested with } \\
\text { microscopy }\end{array}$ & 2.25 & 0.07 & 2.1 & 0.13 & 0.75 & 0.575 & 2.59 & 0.049 & 1.28 & 0.321 \\
\hline $\begin{array}{l}\text { Fever cases } \\
\text { tested with } \\
\text { RDT }\end{array}$ & 34.9 & 0.0000001 & 0.81 & 0.45 & 15.05 & 0.0000001 & 12.86 & 0.0000001 & 15.29 & $0.000000 \mathrm{I}$ \\
\hline $\begin{array}{l}\text { Fever cases } \\
\text { that tested } \\
\text { positive for } \\
\text { malaria with } \\
\text { microscopy }\end{array}$ & 2.36 & 0.06 & 2.44 & 0.09 & 1.13 & 0.378 & 1.71 & 0.166 & 1.61 & 0.227 \\
\hline $\begin{array}{l}\text { Fever cases } \\
\text { that tested } \\
\text { positive for } \\
\text { malaria with } \\
\text { RDT }\end{array}$ & 3.96 & 0.005 & 1.58 & 0.211 & 1.24 & 0.334 & 6.76 & 0.0002 & 0.51 & 0.73 \\
\hline $\begin{array}{l}\text { Confirmed } \\
\text { uncomplicated } \\
\text { malaria cases }\end{array}$ & 7.8 & 0.0000001 & 1.55 & 0.22 & 1.03 & 0.42 & 6.36 & 0.0004 & 0.47 & 0.76 \\
\hline
\end{tabular}

\section{Year-by-year evaluation of fever cases tested with microscopy}

The percent of pregnant women who presented with fever and were tested with microscopy in continuous, year-by-year evaluation

during the study period is illustrated in Figure 2. In the first year of study, the highest proportion of pregnant women who presented with fever and were tested with microscopy was $15.8 \%$. In the second year of study, the proportion was $18.6 \%$ while in the third, fourth and fifth years the proportions were $6.1 \%, 8.0 \%$ and $29.3 \%$ respectively.

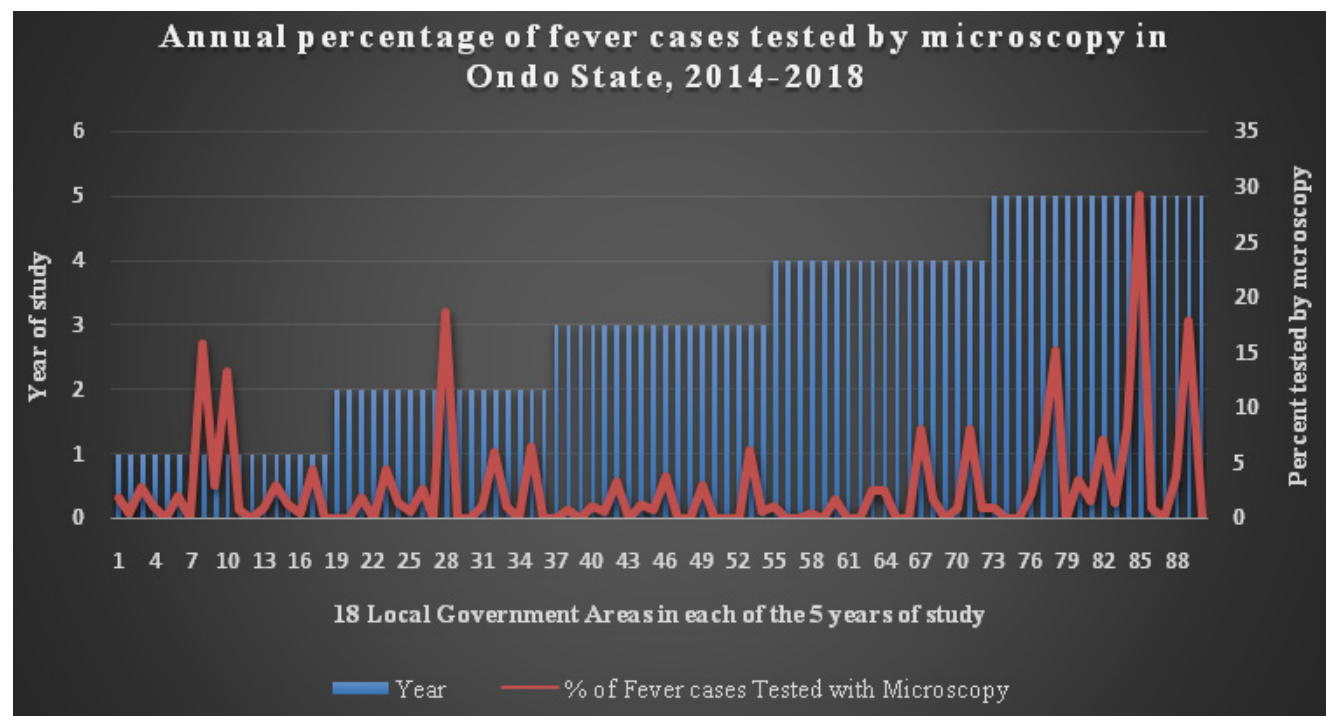

Figure 2 Annual percentage of fever cases tested by microscopy in Ondo State, 20I4-20I8. 


\section{Fever cases tested for malaria parasites with microscopy in the LGAs}

In 2014, only two (11.1\%) LGAs (Idanre and Ilaje) out of 18 LGAs had a relatively high $(15.8 \%$ and $13.2 \%$ respectively) fever cases tested for malaria parasite with microscopy (Figure 3). All other LGAs reported less than 5\% fever cases tested for malaria parasite with microscopy at this period. However, in 2015, 2016 and 2017 the highest LGAs were Ilaje (18.6\%), Ose (8.0\%), Odigbo (8.0\%)while it was also Odigbo LGA (29.3\%) 2018. Throughout the state, the mean $( \pm)$ number of fever cases tested for malaria with microscopy was 2.81 (4.45) in 2014 with a gradual decline to 2.48 (4.51) in 2015 and 1.20 (1.71) in 2016. This mean increased slightly in 2017 to 1.59 (2.46) and finally to 5.47 (7.93) in 2018 without a statistically significant variation (F-statistics $=2.25$; P-value $=0.07$ ) through the years of study (Table 2).

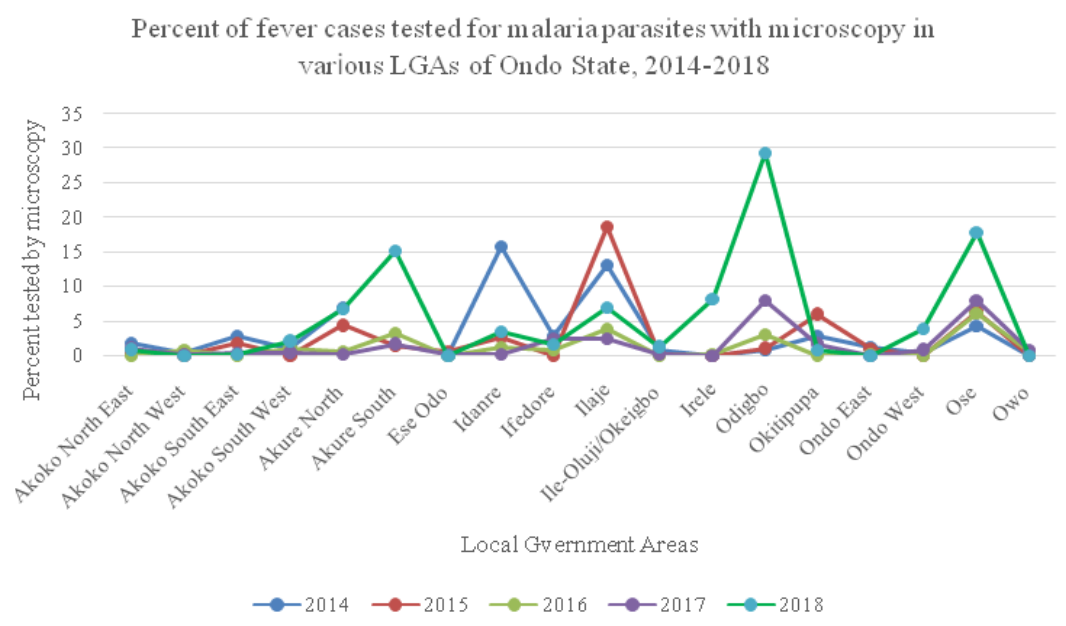

Figure 3 Percent of fever cases tested for malaria parasites with microscopy in various LGAs of Ondo State, 20I4-20I8.

F-statistic $=2.25$, P-value $=0.07$

Table 2 Percent, mean ( $\pm s d)$ and F-statistics of proportions of pregnant women with fever cases who received parasitological test and who tested positive for malaria in the LGAs and in different ecological zones

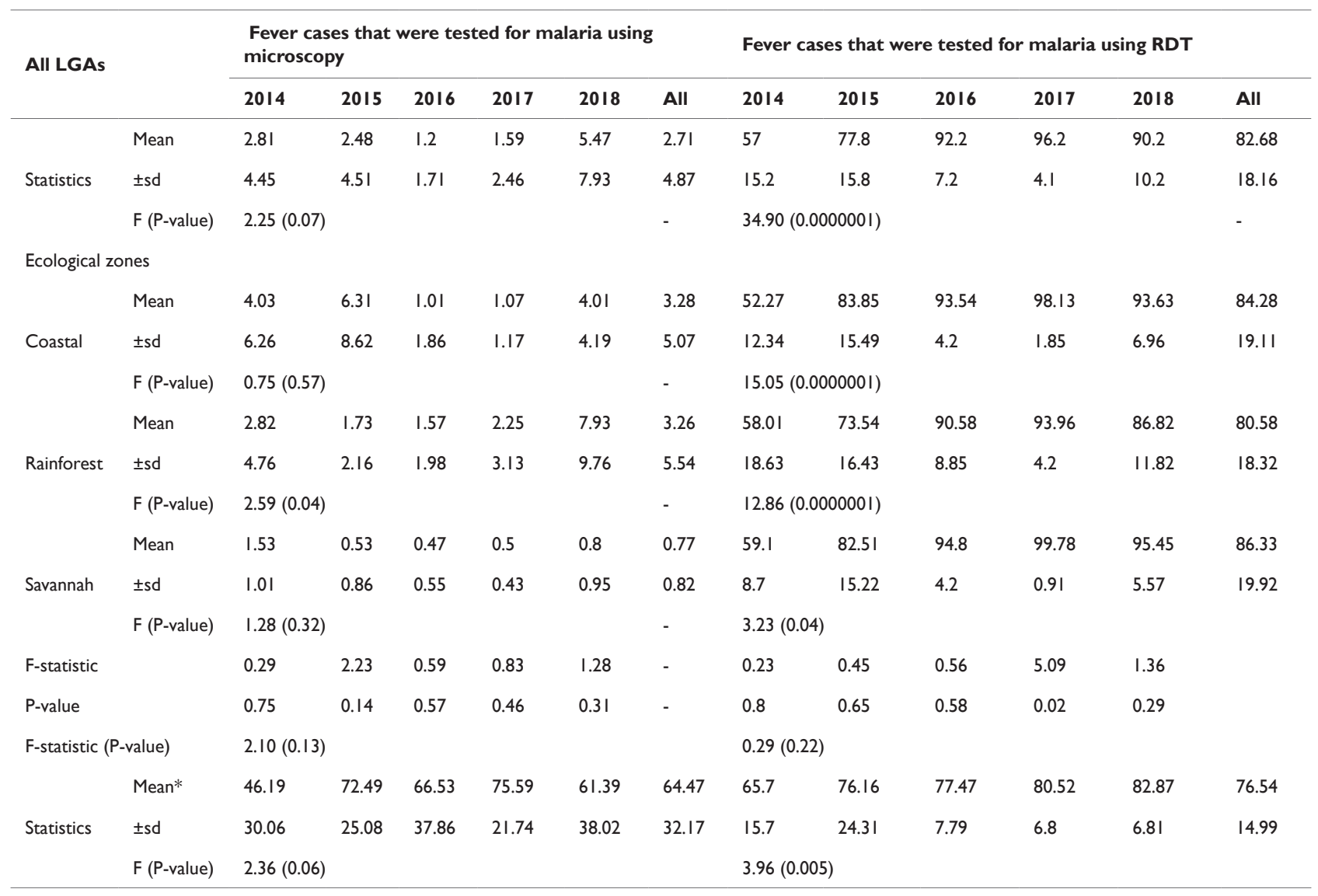


Table Continued.

\begin{tabular}{|c|c|c|c|c|c|c|c|c|c|c|c|c|c|}
\hline \multirow{2}{*}{ All LGAs } & & \multicolumn{6}{|c|}{$\begin{array}{l}\text { Fever cases that were tested for malaria using } \\
\text { microscopy }\end{array}$} & \multicolumn{6}{|c|}{ Fever cases that were tested for malaria using RDT } \\
\hline & & 2014 & 2015 & 2016 & 2017 & 2018 & All & 2014 & 2015 & 2016 & 2017 & 2018 & All \\
\hline \multicolumn{14}{|c|}{ Ecological zones } \\
\hline & Mean! & 68.98 & 79.73 & 97.15 & 84.95 & 53.7 & 76.91 & 66.1 & 72.6 & 77 & 75.4 & 80.4 & 74.31 \\
\hline \multirow[t]{3}{*}{ Coastal } & $\pm s d$ & 46.76 & 18.66 & 3.91 & 24.6 & 40.26 & 31.34 & 12.96 & 11.77 & 9.97 & 7.24 & 2.76 & 9.9 \\
\hline & $F$ (P-value) & $1.13(0.38)$ & & & & & & 1.24 & & & & & \\
\hline & Mean!! & 41.52 & 65.18 & 63.86 & 76.59 & 67.09 & 62.99 & 62.4 & 70.15 & 76.9 & 83.37 & 84.04 & 75.37 \\
\hline \multirow[t]{3}{*}{ Rainforest } & $\pm s d$ & 24.47 & 29.41 & 31.3 & 18.74 & 39.28 & 30.63 & 17.45 & 10.83 & 8.73 & 6.83 & 8.86 & 13.54 \\
\hline & $\mathrm{F}$ (P-value) & I.7I (0.17) & & & & & & 6.76 & & & & & \\
\hline & Mean\# & 33.93 & 83.53 & 33.75 & 63.98 & 54.83 & 55.07 & 73.55 & 94.73 & 79.38 & 78.48 & 82.4 & 81.7 \\
\hline \multirow[t]{2}{*}{ Savannah } & $\pm s d$ & 8.84 & 14.5 & 57.37 & 26.05 & 41.22 & 34.39 & 14.05 & 47.56 & 3.12 & 1.55 & 2.65 & 21.08 \\
\hline & $\mathrm{F}$ (P-value) & $161(0.23)$ & & & & & & 0.51 & & & & & \\
\hline
\end{tabular}

*the mean $( \pm s d)$ proportion of fever cases that tested positive with RDT $(76.54 \pm \mid 4.99)$ was significantly higher $(\mathrm{t}=3 . \mathrm{I} 7, \mathrm{P}$-value $=0.00 \mathrm{I})$ than with microscopy $(64.47 \pm 32.17)$

!!the mean $( \pm \mathrm{sd})$ proportion of fever cases that tested positive with RDT $(75.37 \pm \mid 3.54)$ was significantly higher $(\mathrm{t}=2.55, \mathrm{P}$-value $=0.007)$ than with microscopy $(62.99 \pm 39.28)$

\#the mean $( \pm s d)$ proportion of fever cases that tested positive with RDT $(8 I .7 I \pm 2 I .08)$ was significantly higher $(\mathrm{t}=2.90$, $\mathrm{P}$-value $=0.004)$ than with microscopy $(55.07 \pm 34.39)$

\section{Fever cases tested for malaria with RDT in the LGAs}

In 2014 , the mean $( \pm$ Sd $)$ proportion of pregnant women with fever cases tested for malaria wth RDT was 57.0 (15.2) with Idanre LGA having the highest (91.4\%) and Akure South having the lowest $(23.5 \%)$ proportions respectively. (Figure 4). Subsequently, the mean $( \pm \mathrm{Sd})$ number of fever cases tested for malaria with RDT increased gradually from 77.8 (15.8) in 2015 to 96.2 (4.1) in 2017 before dropping to $90.2(10.2)$ in 2018 with a significant variation (F-statistics $=34.90$, P-value $=0.0000001)$ (Table 2).

\section{Percent of fever cases tested by RDT in various Local Government Areas of Ondo State, 2014-2018}

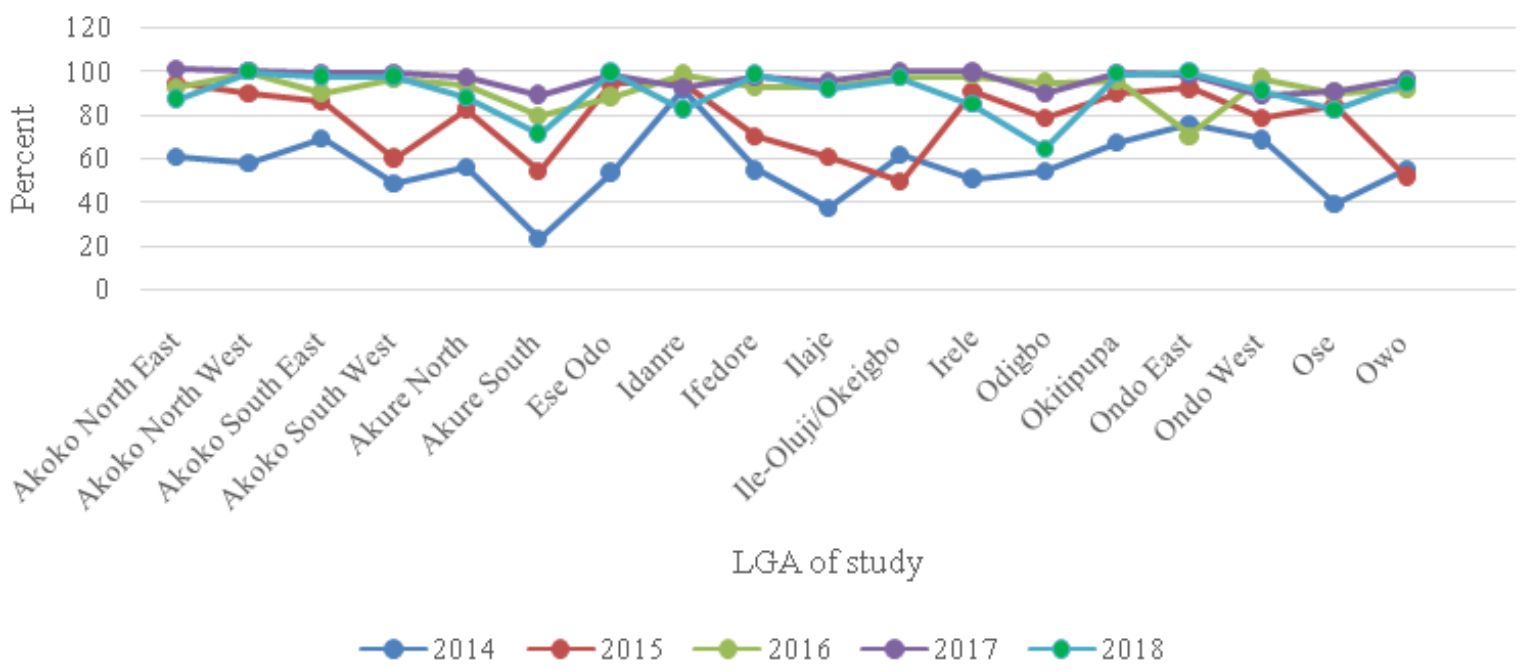

Figure 4 Percent of fever cases tested by RDT in various Local Government Areas of Ondo State, 20I4-20I8.

F-statistic $=34.9, P$-value $=0.0000001$.

\section{Fever cases tested for malaria parasites with microscopy in the ecological zones}

The mean proportion of pregnant women with fever, who were tested for malaria parasites in different ecologizal zones of the state, using microscopy, is as reflected in Figure 5. Throughout the study period, the Savannah ecological zone recorded the lowest mean $( \pm \mathrm{sd})$ proportion of fever cases that tested for malaria parasites at $0.77(0.82)$ while the coastal ecological zone recorded the highest with a mean of 3.28 (5.07) though the difference was not statistically significant. The highest mean proportion of fever cases tested for malaria parasites using microscopy in the Savannah ecological zone was 1.54 in 2014, 
6.31 in the coastal ecological zone in 2015 and 7.93 in the rainforest ecological zone in 2018 (Table 2).

The Table also shows that

a) The mean $( \pm \mathrm{sd})$ proportion of fever cases that tested positive with RDT $(76.54 \pm 14.99)$ was significantly higher $(t=3.17$, $\mathrm{P}$-value $=0.001)$ than the proportion that tested positive with microscopy (64.47 \pm 32.17$)$ in all the LGA, b) The mean $( \pm \mathrm{sd})$ proportion of fever cases that tested positive with RDT $(75.37 \pm 13.54)$ was significantly higher $(\mathrm{t}=2.55$, P-value $=0.007)$ than with microscopy $(62.99 \pm 39.28)$ in the Rainforest ecological zone and

c) The mean $( \pm \mathrm{sd})$ proportion of fever cases that tested positive with RDT $(81.71 \pm 21.08)$ was significantly higher $(t=2.90$, $\mathrm{P}$-value $=0.004)$ than with microscopy $(55.07 \pm 34.39)$ in the Savannah ecological zone.

Mean number of fever cases tested for malaria with microscopy in various ecological zones of Ondo State, 2014-2018

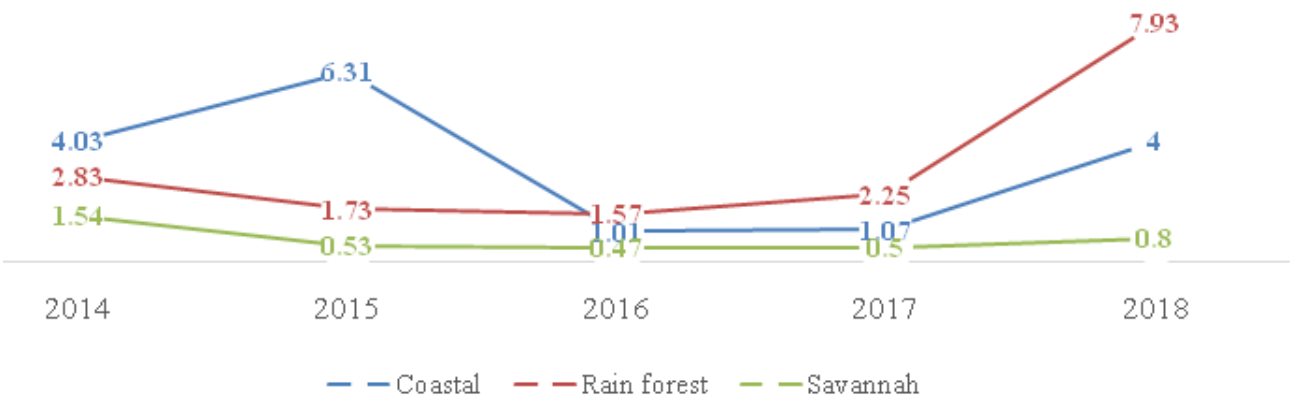

Figure 5 Mean number of fever cases tested for malaria with microscopy in various ecological zones of Ondo State, 2014-2018.

F-statistic $=2$. I0, P-value $=0.13$. There was no overall significant difference in the mean number of women with suspected malaria who had microscopy test for parasite confirmation during the study period.

\section{Fever cases tested for malaria with RDT in the ecological zones}

Fever cases that were tested for malaria using RDT in various ecological zones are illustrated in Figure 6. Although Savannah ecological zone had an overall highest mean of 86.33 (19.92) of fever cases tested for malaria, there was no significant variation when compared with coastal at $84.28(19.11)$ or rainforest (80.58 (18.32) ecological zones. $(\mathrm{F}$-statistic $=0.29$, P-value $=0.22)$.

\section{Mean number of fever cases tested for malaria parasites with RDT in various ecological zones of Ondo State, 2014- 2018}

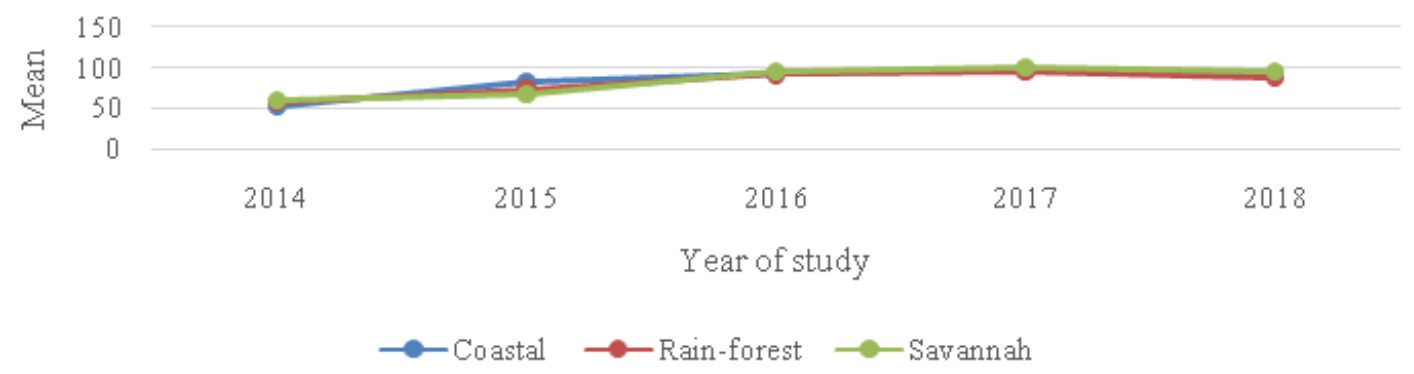

Figure 6 Mean number of fever cases tested for malaria parasites with RDT in various ecological zones of Ondo State, 20I4-20I8.

Overall, the proportion of pregnant women with fever who were tested with RDT from 2014 to 2018 in the different ecological zones were not statistically varied $($ F-staistic $=0.29, \mathrm{P}$-value $=0.22)$.

\section{Fever cases that tested positive for malaria using microscopy or RDT in the LGAs and in ecological zones}

There was a noteworthy variation in the mean $( \pm)$ proportion of fever cases that tested positive for malaria using RDT (F-statistic $=3.96$, $\mathrm{P}$-value $=0.005)$ in all the LGAs which gradually increased from 65.70 (15.70) in 2014 to 82.86 (6.81) in2018. No such significance was observed in fever cases that tested positive for malaria parasites using microscopy (Figure 7). In each year of the study, a higher proportion of fever cases in the LGAs tested positive for malaria using RDT compared to microscopy. However, when the ecological zones were considered, there were no statistical variations in the proportion of fever cases that tested positive for malaria with either microscopy or RDT (Figure 8). 
Mean proportion of fever cases that tested positive for malaria using microscopy or RDT in the LGAs

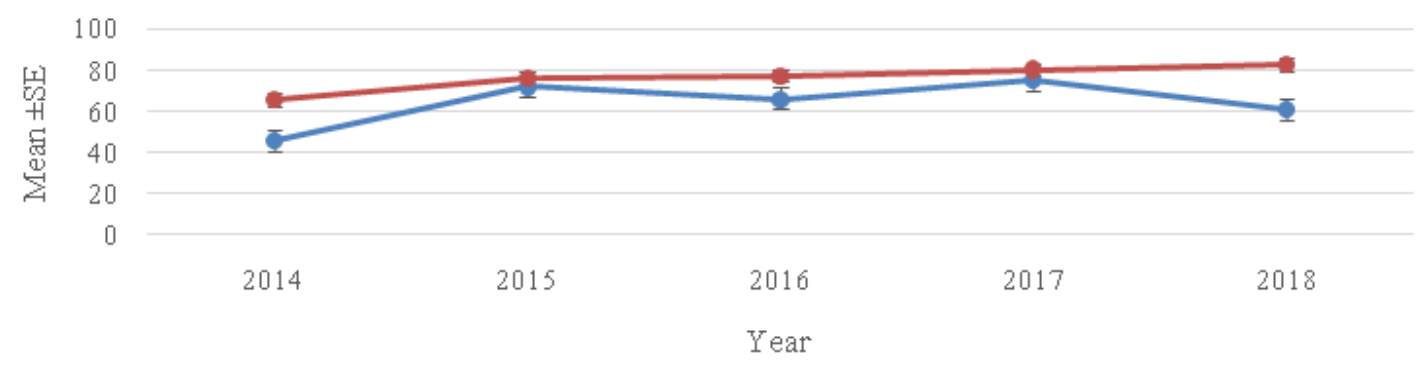

$\longrightarrow$ Microscopy $\longrightarrow$ RDT

Figure 7 Mean proportion of fever cases that tested positive for malaria using microscopy or RDT in the LGAs.

For microscopy, F-statistic=2.36, P-value=0.06; for RDT, F-statistic=3.96, P-value $=0.005$; Pearson's $r=0.160, P$-value $=0.141$.

Mean proportion of fever cases that tested positive for malariausing microscopy or RDT in different ecological zones.

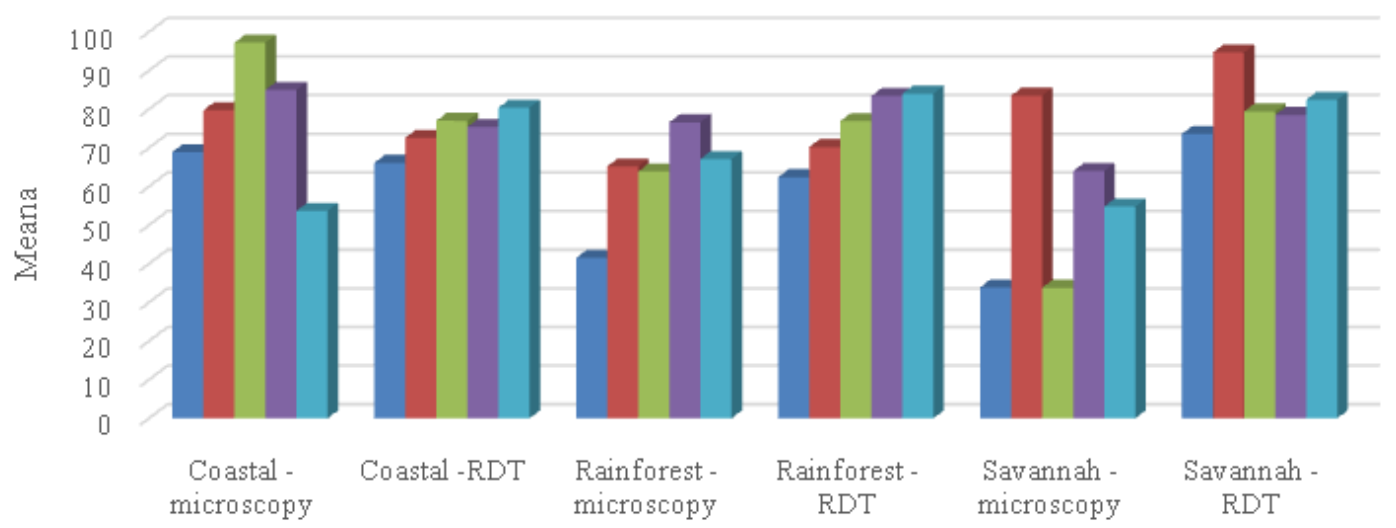

" $2014=2015=2016=2017=2018$

Figure 8 Mean proportion of fever cases that tested positive for malaria using microscopy or RDT in different ecological zones. For microscopy, overall F-statistic $=2.44$, P-value $=0.09$; for $R D T$, overall F-statistic $=1.58, P$-value $=0.21$.

\section{Confirmed uncomplicated malaria cases}

In all the LGAs, the mean proportion of fever cases with confirmed uncomplicated malaria was lowest $(66.03 \pm 16.64)$ in 2014 and highest $(82.46 \pm 8.01)$ in 2018 . Throughout the study period, the overall mean proportion of pregnant women with fever cases that were confirmed as uncomplicated malaria was highest $(81.84 \pm 21.18)$ in the savannah ecological zone without any noteworthy variation with other zones (Table 3).

Table 3 Proportion of pregnant women with confirmed uncomplicated malaria by LGA and by ecological zone of the state

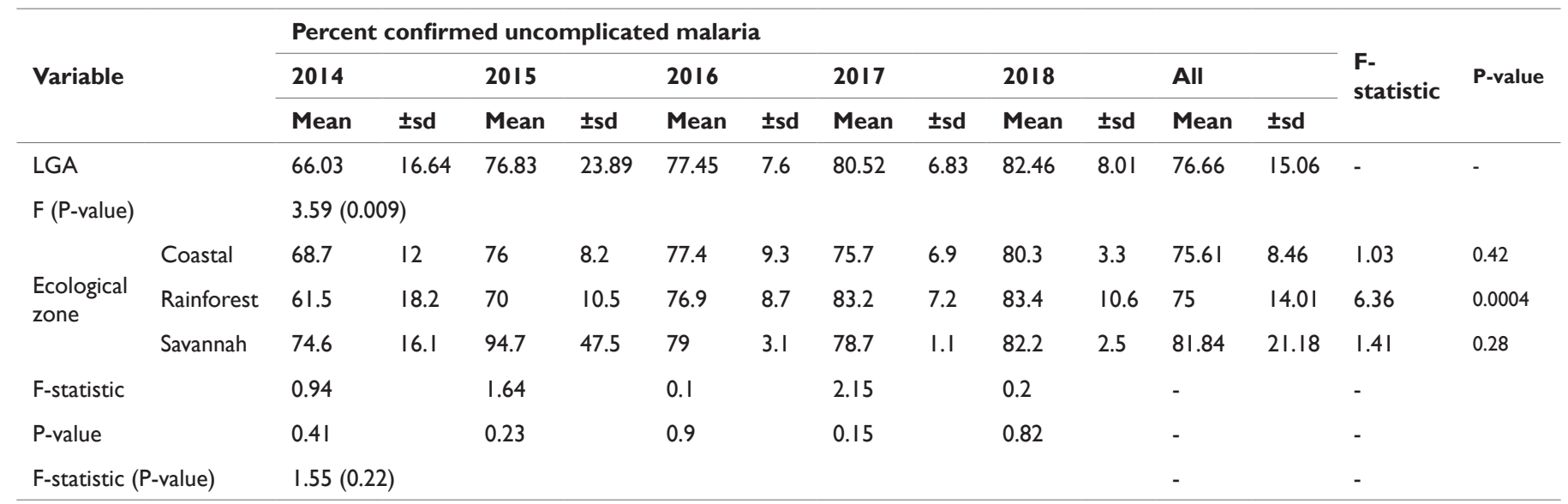




\section{Discussion}

This study of a large database in Ondo State suggests a relatively high prevalence of malaria among pregnant women attending ANCs during the study period. The mean percentages of fever cases positive for malaria by microscopy in $2014(85.6 \%)$ through $2018(61.4 \%)$ were much higher than the $1.4 \%-20 \%$ reported in some Indian studies $^{27-29}$ but similar to the $77.4 \%$ reported from Mozambique. ${ }^{30}$ Similar to the Mozambique study, ${ }^{30}$ less than a third of fever cases in pregnancy had microscopically confirmed malaria parasitaemia. Modern electric microscopy for the diagnosis of malaria in Africa is problematic because,

i. Microscopes are expensive and a large quantity are needed

ii. Accessories for microscopy such as glass slides, fixing alcohol and Giemsa stains are hard to get

iii. Personnel to run the malaria microscopy tests are in short supply, most of them having travelled abroad and those remaining being poorly paid with little incentives

iv. The infrastructure for the microscopy, such as electricity, is not always available

v. The entire health system, such as continuous training, Health Technology Schools, etc, is weak and may not sustain and improve upon malaria microscopy. Thus, it is possible to use RDT as an alternative replacement for microscopic method for malaria diagnosis.

Data from this study also shows that there was no significant correlation (Pearson's r $=0.160$, P-value $=0.141$ ) between fever cases that tested positive using microscopy and fever cases that tested positive using RDT, which did not correspond with the findings of Azikiwe et al which suggested that RDT for the diagnosis of malaria is as reliable as microscopy. ${ }^{31}$ Such discrepancy may be due to different types, methods and sample sizes used in the two studies and does not imply that RDT could not be used as an alternative for microscopy. Additionally, this study identified a significant difference in fever cases tested with microscopy in the rain forest (F-statistics $=2.59$, $\mathrm{P}$-value $=0.049$ ) compared to other ecological zones. This could be due to the species of parasites in different ecological systems of the statemore of falciparum in the rain forest, more of malas on the Atlantic coastline and probably less of falciparum in the Savannah ecological zones. ${ }^{32}$ Continuous entomological studies of malaria vectors and parasites in the different ecological zones of Ondo State will be an added value to better understand the distribution of malaria parasites and clinical presentations among pregnant women in the state.

Among some of the LGAs in this study were some who reported $100 \%$ positivity rate for malaria test, using RDT. This was because pregnant women in these LGAs presented with fever during each ANC visit. This is a positive development because it indicates that many women are now visiting ANC and are being tested for malaria at each visit to avert the deleterious maternal, fetal and neonatal outcomes of malaria. However, it should be noted that malaria is not the only cause of fever in pregnancy. Pregnant women, just like non-pregnant women, are prone to urinary tract infections caused by bacteria such as Escherichi coli, Proteus mirabilis, Klebsiella pneumoniae. ${ }^{33}$ Systemic infections such as appendicitis may be a most common non-obstetric emergency requiring surgery during pregnancy while respiratory infection such as pneumonia may also be cause of fever in pregnancy. ${ }^{33}$
WHO advises that IPTp should be administered through ANC services. ${ }^{17}$ Human factors such as perception of pregnant and nonpregnant women, husband's availability and approval as well as health workers' patient-oriented relation and non-human factors such as distance from health facility, finance and lack of information may be contributory to low compliance with IPTp program. ${ }^{34,35}$

Be that as it may, laboratory over diagnosis of malaria cannot be ruled out in this setting. However, whether a pregnant woman is positive or negative for malaria parasitaemia, SP is still given to sweep the placenta of the parasites. Placental malaria, assessed as late peripheral infections - the most deleterious for the child ${ }^{36}$-should be the main parasitological indicator of the consequencies of gestational malaria ${ }^{37}$ while low birth weight (LBW, defined as $<2500 \mathrm{gm}$ ) a strong predictor of infant health, should be the main clinical indicator. ${ }^{38,39}$

\section{Study limitations}

This study has some limitations which need to be discussed. First, it did not take into consideration the stage of pregnancy or whether the woman was in her first, second or third (or more) pregnancy. Secondly, the study did not consider the quality assurance (QA) of microscopybased diagnosis which is frequently inadequate for ensuring good sensitivity and specificity of malaria diagnosis, adversely affecting health outcomes and optimal use of resources. We assumed that there were (i) a central coordinator in each LGA to oversee quality assurance and that the State Malaria Programmed Manager was the core coordinator for the entire state and (ii) a clear standard operating procedure (SOP) at all levels. However, the State has a robust malaria team which receives regular training by the Federal Ministry of Health and sponsored by international organizations. Also, HIV-status of the pregnant women in the study was not assesses which could have influenced specific information on drug administration patterns such as (i) need for higher doses (ii) possible interference with sulphabased prophylaxis of opportunistic infections. In addition, the supply management and maintenance of microscopes might have needed improvement for more robust study results. Finally, there was no adequate budget as part of funding for malaria case management during the study period.

\section{Conclusion}

Malaria is a health burden in sub-Saharan Africa especially among pregnant women in Ondo State of Nigeria. Between 2014 and 2018, malaria-related indicators including the proportion of pregnant women with fever who were investigated with microscopy and RDT and the proportions that tested positive for malaria, using either of these two methods, were tracked in the state. Data were captured using the District Health Information Management version 2.0 (DHISv2.0). Throughout the study period, the mean $( \pm \mathrm{sd})$ percentage of pregnant women with fever tested for malaria parasites with microscopy in all the LGAs was 2.71 (4.87) (F-statistics=2.25; P-value=0.07) while the overall mean $( \pm \mathrm{sd})$ proportion of pregnat women with fever who were tested for malaria with RDT over the study period was 82.68 (18.16) with a statistically significant variation (F-statistics=34.90; $\mathrm{P}$-value $=0.0000001)$. Overall mean proportion of pregnant women with confirmed fever cases was 76.66 (15.06), mostly in the Savannah ecological system (81.84 (21.18) throughout the study period. Data from this study suggests that RDT gradually replaced microscopy in testing for malaria among pregnant women in Ondo State of Nigeria. It also suggests that some ecological zones may be more underserved than others in assessing malaria in pregnancy. 


\section{Recommendations}

According to Mundy et al., ${ }^{40}$ the challenges for diagnostic laboratories in Africa include defective microscopes, intermittent power, poor consumables and limited time to examine slides. Decision-makers on maternal and child health including pregnancy and delivery should prioritize improvement of hospital laboratories in urban, semi-urban and rural settings, to the extent where their results are as accurate as RDT, though this may not be simple or easy to sustain. ${ }^{41}$ Therefore RDTs should not be out of stock but should be constantly assessed for authenticity so as to provide accurate result for improving diagnosis of malaria in pregnancy quickly and efficiently in malaria endemic sub-Saharan Africa ${ }^{41}$ There should be a focus on

a) Reaching every pregnant women in the state,

b) Sustaining high ANC visits

c) Improving case management of symptomatic pregnant women

d) Evaluating the efficacy and effectiveness of regular screening for malaria parasitemia

e) Directly observed therapy with SP

f) And constantly gathering data on indicators of Malaria in Pregnancy such as LBW, severe anemia and placental malaria. More technical and financial assistance are needed for the State Malaria Elimination Program to improve ANC service utilization.

\section{Acknowledgments}

None.

\section{Funding}

None.

\section{Conflicts of interest}

All authors declare that they have no competing interests.

\section{References}

1. Bellamy C. Globalization and infectious diseases in women. Emerg Infect Dis. 2004;10(11):2022-2024

2. Menendez C, Ordi J, Ismail MR, et al. The impact of placental malaria on gestational age and birth weight. J Infect Dis. 2000;181(5):1740-1745.

3. Rogerson SJ, van den Broek NR, Chaluluka E, et al. Malaria and anemia in antenatal women in Blantyre, Malawi: a twelve-month survey. Am $J$ Trop Med Hyg. 2000;62(3):335-340.

4. Marchant T, Schellenberg JA, Nathan R, et al. Anaemia in pregnancy and infant mortality in Tanzania. Trop Med Int Health. 2004;9(2):262-266.

5. Nosten F, Rogerson SJ, Beeson JG, et al. Malaria in pregnancy and the endemicity spectrum: what can we learn? Trends Parasitol. 2004;20(9):425-432.

6. Shulman CE, Dorman EK. Importance and prevention of malaria in pregnancy. Trans R Soc Trop Med Hyg. 2003;97(1):30-35.

7. Luxemburger C, McGready R, Kham A, et al. Effects of malaria during pregnancy on infant mortality in an area of low malaria transmission. $\mathrm{Am}$ J Epidemiol. 2001;154(5):459-465.

8. Anya SE. Seasonal variation in the risk and causes of maternal death in the Gambia: malaria appears to be an important factor. Am J Trop Med Hyg. 2004;70(5):510-513.
9. Brabin BJ. An analysis of malaria in pregnancy in Africa. Bull World Health Organ. 1983;61(6):1005-1016.

10. Shulman CE, Graham WJ, Jilo H, et al. Malaria is an important cause of anaemia in primigravidae: evidence from a district hospital in coastal Kenya. Trans R Soc Trop Med Hyg. 1996;90(5):535-539.

11. Ndyomugyenyi R, Magnussen P. Anaemia in pregnancy: Plasmodium falciparum infection is an important cause in primigravidae in Hoima district, western Uganda. Ann Trop Med Parasitol. 1999;93(5):457-465.

12. Whitty JM, Edmonds S, Mutabingwa TK. Malaria in pregnancy. Inter J Obst Gynaecol. 2005;112(9):1189-1195.

13. Rogerson SJ, Mkundika P, Kanjala MK. Diagnosis of plasmodium falciparum malaria at delivery: comparison of blood film preparation methods and of blood films with histology. $J$ Clin Microbiol. 2003;41(4):1370-1374.

14. Beeson JG, Cooke BM, Rowe JA, et al. Expanding the paradigms of placental malaria. Trends Parasitol. 2002;18(4):145-147.

15. Patel JC, Hathaway NJ, Parobek CM, et al. Increased risk of low birth weight in women with placental malaria associated with P. falciparum VAR2CSA clade. Sci Rep. 2017;7:7768.

16. WHO. Microscopy. 2018.

17. WHO. Intermittent preventive treatment during infancy with sulphadoxine-pyrimethamine (SP-IPTi) for plasmodium falciparum malaria control in Africa. 2010.

18. Shulman CE, Dorman EK, Cutts F, et al. Intermittent sulphadoxinepyrimethamine to prevent severe anaemia secondary to malaria in pregnancy: a randomised placebo-controlled trial. Lancet. 1999;353(9153):632-636.

19. van Eijk AM, Ayisi JG, ter Kuile FO, et al. Effectiveness of intermittent preventive treatment with sulphadoxine-pyrimethamine for control of malaria in pregnancy in western Kenya: a hospital-based study. Trop Med Int Health. 2004;9(3):351-360.

20. Kayentao K, Kodio M, Newman RD, et al. Comparison of intermittent preventive treatment with chemoprophylaxis for the prevention of malaria during pregnancy in Mali. J Infect Dis. 2005;191(1):109-116.

21. Rogerson SJ, Chaluluka E, Kanjala M, et al. Intermittent sulfadoxinepyrimethamine in pregnancy: effectiveness against malaria morbidity in Blantyre, Malawi, in 1997-99. Trans $R$ Soc Trop Med Hyg. 2000;94(5):549-553.

22. Challis K, Osman NB, Cotiro M, et al. Impact of a double dose of sulphadoxine-pyrimethamine to reduce prevalence of pregnancy malaria in southern Mozambique. Trop Med Int Health. 2004;9(10):1066-1073.

23. Adelusi VA, Folayan W, Akinmoladun OB, et al. Evaluating the impact of preventive interventions against malaria during antenatal care of pregnant women from 2014 to 2018 in Ondo State, Southwest Nigeria. Obstet Gynecol Int J. 2020;11(1):30-37.

24. Afolabi BM, Afolabi TM, Ogunwale A, et al. A 2-month intervention study of preventive clothing against mosquito bites among malnourished and well-nourished children under 5 years of age living on the Atlantic Ocean Coast of Lagos, Southwest Nigeria. Malaria Journal. 2020;19:61.

25. World Health Organization. Baic laboratory methods in medical parasitology. Geneva, Switzerland; 1991.

26. Molineaux L, Gramiccia G. The Garki Project. Research on the epidemiology and control of malaria in Sudan savannah of West Africa. Geneva. Switzerland; 1980:110-129.

27. Singh N, Shukla MM, Sharma VP. Epidemiology of malaria in pregnancy in central India. Bulletin of the World Health Organization. 1999;77(7):567-572. 
28. Sholapurkar SL, Gupta AN, Mahajan RC. Clinical course of malaria in pregnancy--a prospective controlled study from India. Trans $R$ Soc Trop Med Hyg. 1988;82(3):376-379.

29. Singh N, Saxena A, Chand SK, et al. Studies on malaria during pregnancy in a tribal area of central India (Madhya Pradesh). Southeast Asian J Trop Med Public Health. 1998;29:10-17.

30. Bardaji A, Sigauque B, Bruni L, et al. Clinical malaria in African pregnant women. Malaria Journal. 2008;7:27.

31. Azikiwe CCA, Ifezulike CC, Siminialayi IM, et al. A comparative laboratory dignosis of malaria: microscopy versus rapid diagnostic test kits. Asia Pac J Trop Biomed. 2012;2(4):307-310.

32. Gbaye OA, Afolabi OJ, Simon-Oke IA, et al. Abundance and spatial distribution of mosquitoes across three ecological zones of Ondo State, Nigeria. International Journal of Mosquito Research. 2017;4(5):23-27.

33. Maharaj D. Fever in pregnancy: infectious disease \& antimicrobial agents. Antimicrobe; 2020.

34. Hill J, Kazembe P. Reaching the Abuja target for intermittent preventive treatment of malaria in pregnancy in African women: a review of progress and operational challenges. Trop Med Int Health. 2006;11:409-418.
35. Newman R, Moran A, Kayentao K, et al. Prevention of malaria during pregnancy in West Africa: policy change and the power of subregional action. Trop Med Int Health. 2006;11:462-469.

36. Cottrell G, Mary JY, Barro D, et al. Is malaria palcental infection related to peripheral infection at any time of pregnancy? Am J Trop Med Hyg. 2005;73:1112-1118.

37. Briand V, Cottrel G, Massougbodji A, et al. Intermittent preventive treatment for the prevention of malaria during pregnancy in high transmission areas. Malaria Journal. 2007;6:160.

38. McCormick M. The contribution of low birth weight to infant mortality and childhood morbidity. N Eng J Med. 1985;312:82-90.

39. Bloland P, Slutsker L, Steketee R, et al. Rates and risk factors for mortality during the first two years of life in rural Malawi. Am J Trop Med Hyg. 1996;55:82-86.

40. Mundy C, Ngwira M, Kadewele G, et al. Evaluation of microscope conditions in Malawi. Trans R Soc Trop Med Hyg. 2000;94:583-584.

41. Reybum et al. Rapid diagnostic test compared with malaria microscopy. BMJ. 2007;334:403. 